\title{
NOTIFICAÇÕES DE ÓBITOS POR CAUSAS EXTERNAS E VIOLÊNCIA CONTRA IDOSOS: UMA REALIDADE VELADA
}

\author{
Reporting of deaths from external causes and violence against older people: a \\ veiled reality
}

\section{Notificaciones de óbitos de causas externas y la violencia contra mayores: una realidad velada}

\author{
Rubens Correa Meirelles Junior (D) \\ Universidade Federal de Juiz de Fora - UFJF - Governador Valadares - MG - Brasil \\ Julia de Oliveira Castro (iD \\ Universidade Federal de Juiz de Fora - UFJF - Governador Valadares - MG - Brasil \\ Lina Rodrigues de Faria (iD \\ Universidade Federal do Sul da Bahia - UFSB - Itabuna - BA - Brasil \\ Clarice Lima Álvares da Silva (iD \\ Universidade Federal de Juiz de Fora - UFJF - Governador Valadares - MG - Brasil \\ Waneska Alexandra Alves iD \\ Universidade Federal de Juiz de Fora - UFJF - Governador Valadares - MG - Brasil
}

\section{RESUMO}

Objetivo: Descrever o perfil da morbimortalidade da violência contra a pessoa idosa. Métodos: Trata-se de um estudo descritivo e retrospectivo de 14.900 notificações extraídas do Sistema de Informação de Agravos de Notificação (SINAN) e de 18.228 casos do Sistema de Informação de Mortalidade (SIM), de 2012 a 2017, do estado de Minas Gerais, Brasil. Analisaram-se as notificações de violência segundo características das vítimas (sexo, faixa etária, raça/cor e escolaridade), da ocorrência (local, tipo de violência, características do agressor) e as variáveis referentes ao óbito (sexo, faixa etária, raça/cor, estado civil, local de ocorrência e causa do óbito). Resultados: Das notificações analisadas, 54,5\% (8.116) das vítimas eram mulheres, $42,8 \%$ (6.384) brancos e 47,6\% (7.082) com ensino fundamental incompleto/analfabeto. A violência física deu-se em 69,5\% (10.356) dos casos, e o filho apresenta maior frequência no perfil de agressor, com $26,4 \%$ (3.928). Em relação aos óbitos por causas externas, $60,9 \%$ (11.096) eram homens, 37,4\% (6.815) com mais de 80 anos, 54,9\% (10.011) brancos e $37,7 \%(6.867)$ casados. O hospital é o local de óbito mais relatado, $64 \%$ (11.664). Óbitos por outras causas externas: $42,5 \%$ (7.741), sendo as quedas $32,4 \%$ (5.897) do total. Conclusão: As principais vítimas são as mulheres idosas, com baixa escolaridade, e de violência física perpetrada, principalmente, pelo filho, porém aqueles que vêm a óbito com mais frequência são os homens idosos, tendo como principal causa as quedas e os acidentes de transporte.

Descritores: Maus-Tratos ao Idoso; Causas Externas; Sistemas de Informação em Saúde.

\section{ABSTRACT}

Objective: To describe the morbidity and mortality profiles of violence against older people. Methods: This is a descriptive and retrospective study of 14,900 reports made to the Notifiable Disease Information System and 18,228 reports to the Mortality Information System from 2012 to 2017 in the state of Minas Gerais, Brazil. Reports of violence were analyzed considering characteristics of the victim (sex, age group, race/color and education) and violence (place of occurrence, type of violence, characteristics of the perpetrator) and death-related variables (sex, age group, race/color, marital status, place of occurrence and cause of death). Results: $54.5 \%(8,116)$ of the reports referred to women, $42.8 \%(6,384)$ to white victims and $47.6 \%(7,082)$ to victims who were either illiterate or had not completed primary education. Physical violence accounted for 69.5\% (10,356) of the cases and children were the main perpetrators, representing $26.4 \%(3,928)$ of the cases. $60.9 \%(11,096)$ of the reports of death from external causes referred to men, $37.4 \%(6,815)$ to people over 80 years old, $54.9 \%(10,011)$ to white people and $37.7 \%$

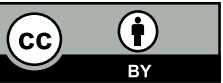


$(6,867)$ to married people. The hospital was the place where most of the deaths occurred, representing $64 \%(11,664)$ of the cases. Deaths from other external causes were: $42.5 \%(7,741)$, with falls accounting for $32.4 \%(5,897)$ of the total. Conclusion: The main victims were older women with poor education and who experienced physical violence perpetrated by the child. However, deaths were more common among older men and were mainly caused by falls and other transport accidents.

Descriptors: Elder Abuse; External Causes; Health Information Systems.

\section{RESUMEN}

Objetivo: Describir el perfil de la morbimortalidad de la violencia contra el mayor. Métodos: Se trata de un estudio descriptivo y retrospectivo de 14.900 notificaciones del Sistema de Información de Agravios de Notificación (SINAN) y de 18.228 casos del Sistema de Información de Mortalidad (SIM) entre 2012 y 2017 en el estado de Minas Gerais, Brasil. Se analizaron las notificaciones de violencia según las características de las victimas (sexo, franja de edad, raza/color y escolaridad), de la ocurrencia (local, tipo de violencia, características del agresor) y las variables del óbito (sexo, franja de edad, raza/color, estado civil, local de ocurrencia y causa del óbito). Resultados: De entre las notificaciones analizadas el 54,5\% (8.116) de las víctimas eran mujeres, el 42,8\% eran (6.384) blancos y el 47,6\% (7.082) tenían educación primaria incompleta/analfabeto. La violencia física se dio en el $69,5 \%$ (10.356) de los casos y el hijo tiene el perfil de agresor en el 26,4\% (3.928) de ellos. Respecto los óbitos de causas externas el $60,9 \%$ (11.096) eran hombres, el 37,4\% (6.815) tenían más de 80 años, el 54,9\% (10.011) eran blancos y el 37,7\% (6.867) eran casados. El hospital es el sitio de óbito más relatado en el 64\% (11.664) de los casos. El 42,5\% (7.741) fueron óbitos de otras causas externas con el 32,4\% (5.897) de ellos, por caídas. Conclusión: Las principales víctimas son las mujeres mayores, de baja escolaridad y de violencia física practicada principalmente por el hijo, sin embargo, los que se mueren con más frecuencia son los hombres mayores que tienen las caídas y los accidentes de transporte como causa principal.

Descriptores: Maltrato al Anciano; Causas Externas; Sistemas de Información en Salud.

\section{INTRODUÇÃO}

O envelhecimento demográfico é um fenômeno verificado por meio do aumento na proporção de pessoas idosas (60 anos e mais) em relação à população em geral. No Brasil, o processo de transição demográfica apresentou início com a redução das taxas de fecundidade e mortalidade, com a modificação do perfil de morbimortalidade, isto é, com a redução da incidência de óbitos por doenças infecciosas e parasitárias e com o aumento das doenças crônico-degenerativas não transmissíveis ${ }^{(1)}$. Cabe enfatizar, ainda, a longevidade, a urbanização acelerada e a maior participação econômica das mulheres ${ }^{(2)}$.

A composição etária do Brasil vem apresentando constante aumento da proporção de idosos: em 2012, estimouse 25,4 milhões de pessoas com idade a partir de 60 anos, enquanto que, em 2017, esse contingente populacional chegou à soma de 30,2 milhões. Portanto, o número de idosos cresceu $18,8 \%$ em 5 anos, chegando a representar $14,7 \%$ da população brasileira em $2017^{(3)}$.

Um número significativo de idosos brasileiros está em plena atividade social e econômica(4), assumindo papéis diferentes na comunidade, na família e entre os amigos. O aumento da sobrevida está intimamente relacionado ao desenvolvimento de novas tecnologias e à melhoria do acesso à saúde e à seguridade social. Contudo, o aumento da idade tem influência direta na elevação da vulnerabilidade física e mental dessa população, tornando-a vítima em potencial da violência(5).

Em 2001, validou-se o termo maus-tratos contra idosos como ações únicas ou repetidas que causam sofrimento ou angústia, ou, ainda, a ausência de ações que são devidas, que ocorrem numa relação em que haja expectativa de confiança ${ }^{(6)}$. Em paralelo, é definido como um ato que pode se caracterizar de natureza física ou psicológica, de ordem financeira ou material, e por omissão por parte da família e/ou profissionais de saúde. Seja intencional, seja involuntário, sempre resulta em sofrimento desnecessário, lesão ou dor, perda ou violação dos direitos humanos, e provoca uma redução na qualidade de vida do idoso(7).

A violência contra os idosos é uma importante demanda, que tem acompanhado o crescimento dessa população, acarretando adoecimento físico e psicológico (depressão, desordem pós-traumática, agitação, fadiga, perda de identidade e tentativas de suicídio) ${ }^{(8)}$. Entre as vivenciadas pelos idosos, a violência por causas externas e sua repercussão na saúde têm sido objeto de inúmeras pesquisas no campo da saúde pública, demonstrando tratar-se de fenômeno de alta prevalência e grande impacto na saúde mental e física. Cabe destacar o impacto das violências doméstica, sexual, verbal e física (sendo a última caracterizada como o uso de força física que pode resultar em dano) $)^{(9,10)}$. 
A violência impacta a qualidade de vida dos idosos, podendo ter graves efeitos e resultando em problemas psicológicos, financeiros, sociais e físicos, e na incapacidade perene, permanente ou a morte da pessoa idosa(11). A incapacidade pode, por sua vez, aumentar a dependência funcional do idoso, aumentando o risco da violência física e de outros tipos de abuso por terceiro ${ }^{(12)}$. A temática é desafiadora para a saúde pública, principalmente para ações de controle e prevenção, devido a sua multicausalidade ${ }^{(7,11)}$.

Dentre as consequências da violência, uma das mais graves, é a morte do indivíduo violentado, devendo esse óbito entrar nas causas externas de acordo com a $10^{a}$ edição da Classificação Internacional de Doença (CID- 10). As causas externas são consideradas agravos de acidentes não intencionais e agravos resultantes de lesões intencionalmente provocadas em si próprio ou em outro, sendo agrupadas em violência e acidentes ${ }^{(13)}$. Esses agravos são considerados eventos evitáveis, sendo utilizados como indicadores e determinantes da saúde de uma população(14).

A violência contra idosos é um fenômeno de notificação recente no mundo e, especificamente, no Brasil. Contra a pessoa idosa emerge, nesse início de século, como um grave e crescente problema de saúde pública, interferindo em diferentes esferas da qualidade de vida das pessoas ${ }^{(15)}$. As produções acadêmicas brasileiras em torno desse fenômeno apresentaram o seu boom a partir dos anos 2000, com a ampliação dos debates em diversas áreas da saúde em função do impacto social e na saúde ${ }^{(10)}$. Infelizmente, os inúmeros abusos cometidos são subnotificados, não revelando a magnitude desse fenômeno ${ }^{(16,17)}$. Nesse sentido, o presente estudo objetivou descrever o perfil da morbimortalidade da violência contra a pessoa idosa.

\section{MÉTODOS}

Estudo descritivo realizado com dados secundários, retrospectivos sobre causas externas, obtidos nos Sistemas de Informação de Agravos de Notificação - versão Net (SINAN-Net) e de Mortalidade (SIM), disponibilizados publicamente pelo Secretaria Estadual de Saúde de Minas Gerais (MG), Brasil, abrangendo as notificações de violência doméstica, sexual e/ou outras violências na população idosa residente em MG.

Diante do cenário de crescente aumento da violência e da mortalidade na população selecionada para análise, observou-se a necessidade de estudos mais recentes para avaliar se o crescimento do número de casos continua a ocorrer na região. Por essa mesma razão, optou-se pelo período de tempo de 2012 (último ano dos estudos anteriores) a 2017 (dados mais recentes disponíveis no sistema TABNET). De forma mais específica, os dados selecionados para análise englobam o período de 01 de janeiro de 2012 a 31 de dezembro de 2017.

Um estudo realizado no estado de MG demonstrou que, de 1999 a 2008, ocorreu um crescimento da mortalidade por causas externas em MG ${ }^{(18)}$. Outro estudo, analisando o período de 2011 a 2012, demonstra que as notificações de violência contra os idosos de MG duplicou ${ }^{(19)}$.

Para a seleção da faixa etária, utilizou-se a definição de idoso conforme a Política Nacional da Saúde da Pessoa Idosa (PNSPI), que define como pessoa que tem 60 anos ou mais de idade ${ }^{(20)}$. Optou-se por usar essa definição no estudo por ser a PNSPI um documento recente e importante sobre a pessoa idosa no Brasil. Captaram-se os dados do SINAN-Net por meio do Portal de Vigilância e Proteção à Saúde da Secretaria de Estado de Saúde de MG, tendo sido coletados no ano de 2018.

Analisaram-se as variáveis disponíveis na ficha de notificação/investigação que permitissem caracterizar os casos notificados de violência contra idosos, sendo elas: características demográficas das vítimas (sexo, raça/cor da pele, escolaridade), características da ocorrência (local, violência de repetição, evolução), tipo de violência e características do agressor (vínculo com a vítima).

Quanto aos dados sobre mortalidade, obtiveram-se os dados do SIM a partir da mesma fonte que o SINANNet, e as causas básicas dos óbitos estão agrupadas de acordo com a classificação proposta no CID-10 (Código Internacional de Doenças): acidentes de transporte (V01-V99); outras causas externas de lesões acidentais (W00-X59); lesões autoprovocadas voluntariamente (X60-X84); agressões (X85-Y09); eventos cuja intenção é indeterminada (Y10-Y34); complicações de assistência médica e cirúrgica (Y40-Y84) e sequelas causas externas de morbidade e mortalidade (Y85-Y89)(11). Buscou-se, também, a caracterização dos óbitos segundo as variáveis disponíveis: sexo, faixa etária, raça/cor, estado civil, local de ocorrência e causa do óbito(18).

Processou-se a análise estatística pelo software Epilnfo 7.2, com cálculos de proporções, médias e taxas de incidência. No cálculo das taxas de incidência para os anos de estudo, no numerador encontra-se o produto do número de casos novos de uma doença ocorridos em uma população em um determinado período, que é 
multiplicado por 100.000; no denominador, o número de pessoas sob risco de desenvolver a mesma doença durante o mesmo período. A população residente é definida pelas projeções adotadas pelo Instituto Brasileiro de Geografia e Estatística (IBGE)(3) ou seja, de projeção da tendência do crescimento demográfico. Também se calculou as taxas de mortalidade específica entre os casos internados. Para a mortalidade específica, tem-se por numerador a soma do número de óbitos ocorrido durante o período de 2012 a 2017, multiplicado por 100 mil habitantes, e a soma da população residente durante o mesmo período por denominador ${ }^{(19)}$.

O estudo dispensou parecer do Comitê de Ética em Pesquisa (CEP) por utilizar dados secundários, não nominais e de domínio público, conforme resolução do Conselho Nacional de Saúde (CNS) de número 510/2016. Contudo, realizou-se o estudo em consonância com os requisitos da Resolução CNS n. 466/2012.

\section{RESULTADOS}

Identificaram-se 14.900 notificações de violência contra idosos nos estabelecimentos notificadores do SINANNet no período analisado. A média é de $2.483,3$ casos notificados por ano, e houve um aumento de $276,90 \%$ nas notificações quando comparados os anos de 2012 e 2017. As taxas de incidência (por 100 mil habitantes), para o período de 2012 a 2017, equivalem a 50,2; 73,4; 93,5; 113,9; 105,4 e 113,6 casos, respectivamente.

O sexo feminino apresentou taxas de incidência maiores quando comparado ao sexo masculino conforme apresenta a Figura 1.

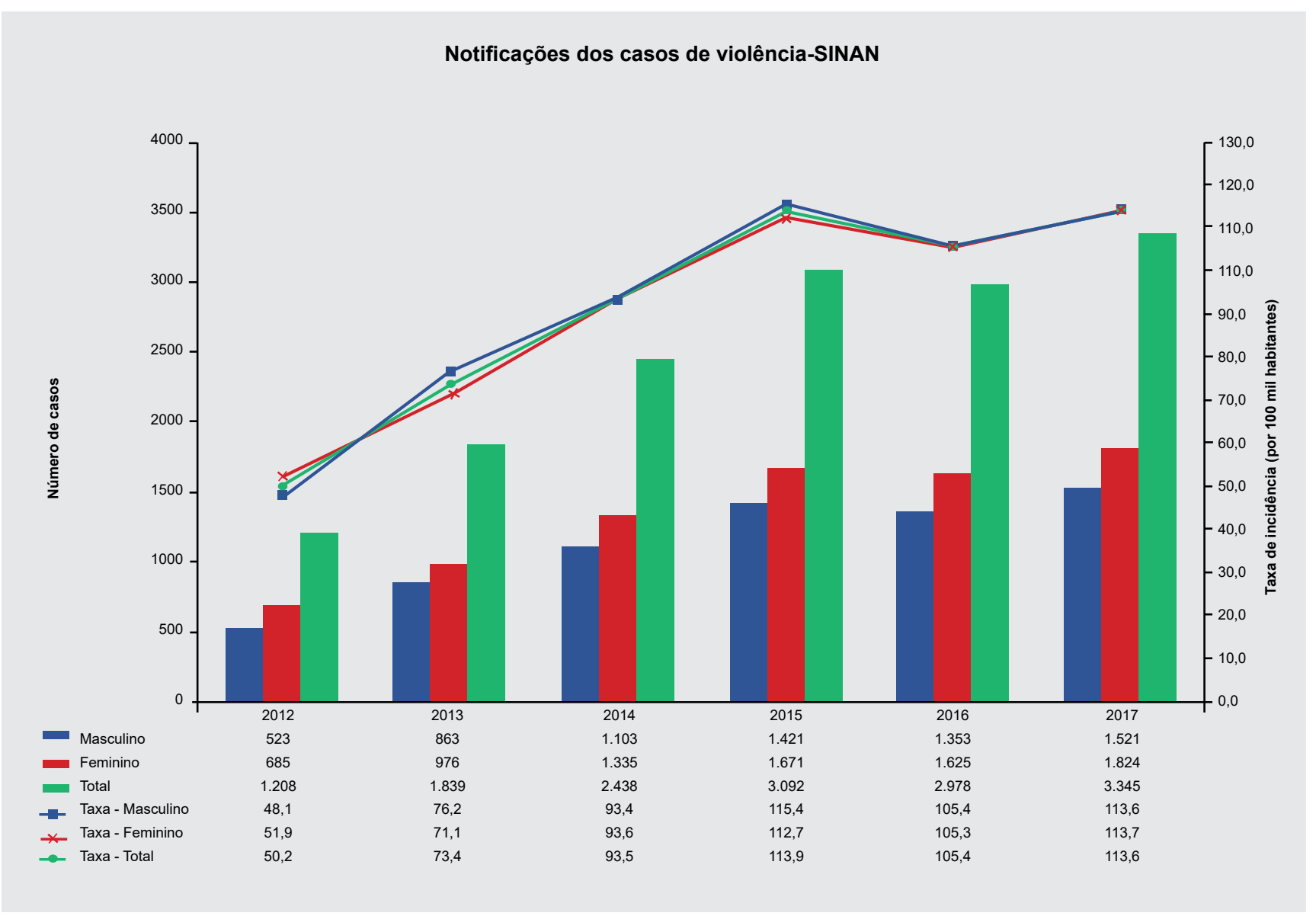

Fonte: SINAN - Sistemas de Informação de Agravos de Notificação/Ministério da Saúde (TABNET).

Figura 1 - Distribuição da ocorrência de casos de violência e taxas de incidência (por 100 mil habitantes) em idosos, por sexo. Minas Gerais, Brasil, 2012 a 2017.

Do total de casos notificados, $54,5 \%$ referiam-se a vítimas do sexo feminino e $45,5 \%$ a do sexo masculino, sendo que, destes últimos, a maior parte (42,8\%) declarou-se branco. Dos idosos acometidos de violência, 47,3\% apresentavam ensino fundamental incompleto/analfabeto, porém $41,9 \%$ das notificações apresentaram esse campo ignorado ou deixado em branco, como consta na Tabela I. 
Tabela I - Distribuição dos casos de violência em idosos, por variáveis sociodemográficas e epidemiológicas. Minas Gerais, Brasil, 2012 a 2017.

\begin{tabular}{|c|c|c|c|}
\hline Variável & & $\mathbf{n}$ & $\%$ \\
\hline \multirow[t]{2}{*}{ Sexo } & Feminino & 8116 & 54,5 \\
\hline & Masculino & 6784 & 45,5 \\
\hline \multirow[t]{6}{*}{ Raça } & Branca & 6384 & 42,8 \\
\hline & Parda & 5064 & 34,0 \\
\hline & Ignorado & 1682 & 11,3 \\
\hline & Preta & 1613 & 10,8 \\
\hline & Amarela & 111 & 0,7 \\
\hline & Indígena & 46 & 0,3 \\
\hline \multirow[t]{8}{*}{ Escolaridade } & Analfabeto & 1888 & 12,7 \\
\hline & Ensino fundamental incompleto & 5194 & 34,9 \\
\hline & Ensino fundamental completo & 516 & 3,5 \\
\hline & Ensino médio incompleto & 277 & 1,9 \\
\hline & Ensino médio completo & 565 & 3,8 \\
\hline & Educação superior incompleta & 37 & 0,3 \\
\hline & Educação superior completa & 186 & 1,2 \\
\hline & Ignorado & 6237 & 41,9 \\
\hline \multirow[t]{7}{*}{ Local de ocorrência } & Residência & 10889 & 73,1 \\
\hline & Via pública & 1600 & 10,7 \\
\hline & Bar ou Similar & 298 & 2,0 \\
\hline & Habitação Coletiva & 104 & 0,7 \\
\hline & Escola & 31 & 0,2 \\
\hline & Outros & 568 & 3,8 \\
\hline & Ignorado & 1409 & 9,5 \\
\hline \multirow[t]{7}{*}{ Tipo de violência } & Violência Física & 10356 & 69,5 \\
\hline & Violência de repetição & 5505 & 36,9 \\
\hline & Violência Psicológica/moral & 4870 & 32,7 \\
\hline & Negligência/Abandono & 2321 & 15,6 \\
\hline & Violência Financeira & 905 & 6,1 \\
\hline & Tortura & 579 & 3,9 \\
\hline & Violência sexual & 336 & 2,3 \\
\hline \multirow[t]{10}{*}{ Perfil do Agressor } & Filho(a) & 3928 & 26,4 \\
\hline & Desconhecido(a) & 1948 & 13,1 \\
\hline & Amigos/conhecidos & 1874 & 12,6 \\
\hline & Conjugue & 1458 & 9,8 \\
\hline & Irmão & 453 & 3,0 \\
\hline & Cuidador(a) & 263 & 1,8 \\
\hline & Ex-cônjuge & 179 & 1,2 \\
\hline & Namorado(a) & 92 & 0,6 \\
\hline & Ex-Namorado(a) & 48 & 0,3 \\
\hline & Outros Vínculos & 1766 & 11,9 \\
\hline
\end{tabular}

Fonte: SINAN: Sistemas de Informação de Agravos de Notificação; Ministério da Saúde (TABNET)

Em relação ao perfil de violência (local de ocorrência, tipo de violência e a relação do agressor com a vítima), o que se pode observar é que a maioria dos episódios contra idosos ocorreram no domicílio $(73,1 \%)$, seguido da via pública $(10,7 \%)$ e um número importante de ocorrências apresentou local ignorado $(9,5 \%)$. 
Como tipos de violência mais relatados estão o abuso físico (69,5\%), violência psicológica (32,7\%) e o abandono $(15,6 \%)$. Em menor proporção, casos de violência financeira $(6,1 \%)$, tortura $(3,9 \%)$ e abuso sexual $(2,3 \%)$. Conforme disposto na Tabela I, também é possível observar que muitos casos eram de repetição $(36,9 \%)$, ou seja, os idosos vêm sofrendo com essa questão de forma contínua.

Em relação aos agressores, esses eram, em sua maioria, filhos $(26,4 \%)$, pessoas desconhecidas $(13,1 \%)$, amigos/conhecidos $(12,6 \%)$ e cônjuges $(9,8 \%)$. Dentre os agressores menos citados estão o/a ex-cônjuge $(1,2 \%)$, namorado/a $(0,6 \%)$ e ex-namorado/a $(0,3 \%)$.

Em relação aos óbitos por causas externas em MG, registrou-se 18.228 óbitos de idosos por causas externas no período de 2012 a 2017, com aumento de 132,5\% em 2017 em comparação a 2012, representando uma média de 3.038 óbitos/ano e 8,33 óbitos/dia, conforme disposto na Figura 2.

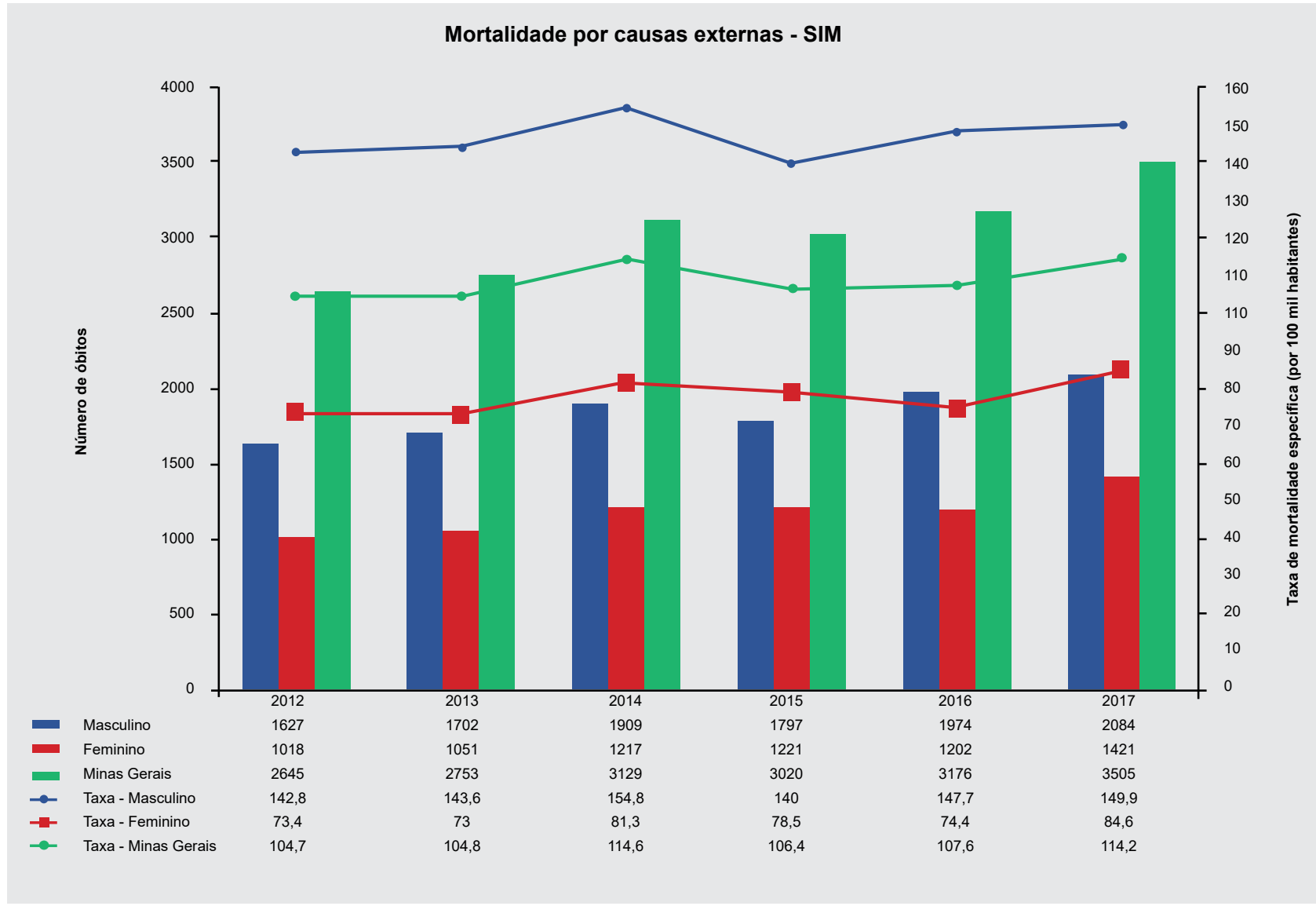

Fonte: SIM: Sistema de Informações sobre Mortalidade; Ministério da Saúde

Figura 2 - Distribuição da ocorrência de óbitos por violência e taxas de mortalidade (por 100 mil habitantes) em idosos, por sexo. Minas Gerais, Brasil, 2012 a 2017.

As taxas de mortalidade específica por causas externas (por 100 mil habitantes) variaram, de 2012 a 2017, de 104,7 óbitos a 114,2 óbitos, quando a taxa de mortalidade específica média é de 108,7 óbitos por 100 mil habitantes. Entre os idosos do sexo feminino, a taxa representa 73,4 óbitos em 2012 e 84,6 óbitos em 2017, com uma média de 77,5 óbitos por 100 mil habitantes. No que tange às pessoas do sexo masculino, em 2012, o valor de taxa é de 142,8 óbitos e, em 2017, 149,9 óbitos, com a média de 146,4 óbitos por 100 mil habitantes.

Em relação ao sexo, os idosos do sexo masculino equivalem a $60,9 \%$ dos óbitos registrados. As faixas etárias que apresentaram maior frequência de óbitos são as de 60 a 69 anos e 80 anos ou mais ( $34,7 \%$ e $37,4 \%$ respectivamente). A cor branca representa a maioria dos óbitos $(54,9 \%)$, seguida da parda $(32,5 \%)$. Quanto ao estado civil, entre os casados e viúvos houve maior número de óbitos $(37,7 \%$ e $28,4 \%$, respectivamente). Dentre os locais de óbito, estão: os hospitais $(64,0 \%)$, domicílios $(14,0 \%)$ e vias públicas $(11,6 \%)$, conforme disposto na Tabela II.

No tocante às causas de óbito, segundo a CID10, as lesões por acidentes representam a primeira causa $(42,5 \%)$, sendo que, nessa categoria, as quedas representam $32,4 \%$ do total de casos e os acidentes de transporte representam $22,8 \%$ dos óbitos. As categorias que sucedem os acidentes são eventos indeterminados $(17,0 \%)$, agressões $(6,7 \%)$ e lesões autoprovocadas $(6,6 \%)$ (Tabela II). 
Tabela II - Distribuição dos óbitos por violência em idosos, por variáveis sociodemográficas e epidemiológicas. Minas Gerais, Brasil, 2012 a 2018.

\begin{tabular}{|c|c|c|c|}
\hline Variáveis & & $\mathbf{n}$ & $\%$ \\
\hline \multirow[t]{2}{*}{ Sexo } & Masculino & 11096 & 60,9 \\
\hline & Feminino & 7132 & 39,1 \\
\hline \multirow[t]{6}{*}{ Raça/Cor } & Branca & 10011 & 54,9 \\
\hline & Parda & 5920 & 32,5 \\
\hline & Preta & 1120 & 6,1 \\
\hline & Amarela & 45 & 0,2 \\
\hline & Indígena & 15 & 0,1 \\
\hline & Ignorado & 1117 & 6,1 \\
\hline \multirow[t]{3}{*}{ Faixa etária } & 60 a 69 anos & 6328 & 34,7 \\
\hline & 70 a 79 anos & 5085 & 27,9 \\
\hline & 80 anos ou mais & 6815 & 37,4 \\
\hline \multirow[t]{6}{*}{ Estado Civil } & Casado & 6867 & 37,7 \\
\hline & Viúvo & 5174 & 28,4 \\
\hline & Solteiro & 2640 & 14,5 \\
\hline & Separado judicialmente & 1284 & 7 \\
\hline & União consensual & 219 & 1,2 \\
\hline & Ignorado & 2044 & 11,2 \\
\hline \multirow[t]{6}{*}{ Local de ocorrência } & Hospital & 11664 & 64 \\
\hline & Domicílio & 2550 & 14 \\
\hline & Via Pública & 2120 & 11,6 \\
\hline & Outros & 1075 & 5,9 \\
\hline & Outro Estabelecimento de Saúde & 768 & 4,2 \\
\hline & Ignorado & 51 & 0,3 \\
\hline \multirow[t]{9}{*}{ Causa de óbitos CID-10 } & Outras causas externas de lesões acidentes & 7751 & 42,5 \\
\hline & Quedas & 5897 & 32,3 \\
\hline & Acidentes de transporte & 4174 & 22,9 \\
\hline & Eventos cuja intenção é indeterminada & 3098 & 17 \\
\hline & Agressões & 1229 & 6,7 \\
\hline & Lesões autoprovocadas voluntariamente & 1198 & 6,6 \\
\hline & Intervenções legais e operações de guerra & 2 & 0,01 \\
\hline & Complicações da assistência médica e cirúrgica & 673 & 3,7 \\
\hline & Sequelas de causas externas & 103 & 0,6 \\
\hline
\end{tabular}

Fonte: SIM: Sistema de Informações sobre Mortalidade; Ministério da Saúde (TABNET); CID-10: Código Internacional de Doenças

\section{DISCUSSÃO}

Na presente pesquisa os dados do SINAN revelaram que a violência contra o idoso ocorre, predominantemente, na população feminina, branca e com até 4 anos de estudo, principalmente no domicílio, tendo o/a filho/a como o agressor mais presente e a violência física como a mais relatada.

Em relação ao perfil de mortalidade dos idosos, pelos dados do SIM, no período analisado predominam os óbitos entre os indivíduos do sexo masculino, brancos, casados/viúvos, no sistema hospitalar e decorrente de quedas ou acidentes de trânsito principalmente. Assim, mostrando um quadro preocupante do aumento da ocorrência dos casos, porém sem grande aumento das taxas de mortalidade. Outros estudos corroboram para a percepção do aumento de óbitos na população idosa, principalmente na população masculina ${ }^{(18,19,21)}$. 
Acerca dos casos de violência contra idosos notificados em MG, nota-se que existe uma incidência maior entre as mulheres idosas, sendo elas vítimas das mais variadas agressões, assim como dados de pesquisas recentes acerca do tema ${ }^{(22-25)}$. Estudos internacionais têm demonstrado associação do processo de fragilização e abusos praticados contra os idosos ${ }^{(22,26)}$ com fatores de risco para a violência, a depressão e o estresse ${ }^{(26)}$.

Os dados recolhidos na presente pesquisa apontam que idosos com até 4 anos de estudo incompletos são os mais violentados, porém não se pode afirmar que a baixa escolaridade está relacionada com a maior propensão. Um estudo que propôs analisar os dados de violência do SINAN-Net identificou que, em 11.297(60,9\%) casos de notificações no Brasil, os idosos apresentavam ensino fundamental incompleto ${ }^{(25)}$. Um estudo internacional revelou que o nível de escolaridade das mulheres está intimamente relacionado às situações de violência ${ }^{(12)}$.

Em relação à localização da violência, na presente pesquisa, o domicílio se apresenta como o local mais apontado. A violência no ambiente domiciliar possui relação com o número de pessoas que moram na mesma casa e, principalmente, com a quantidade de gerações ${ }^{(27)}$.

Na presente análise identificou-se a violência física (75,6\%) como o abuso mais recorrente entre os idosos. Estudo realizado no Distrito Federal evidenciou a violência psicológica em primeiro lugar nos casos notificados, seguida pela violência física, com um aumento do número de denúncias, mais expressivas no sexo masculino ${ }^{(28)}$. Em uma meta-análise verificou-se a prevalência de violência física $(2,6 \%)$ em posição inferior à psicológica (11,6\%), financeira $(6,8 \%)$ e à negligência $(4,2 \%)$ em idosos ${ }^{(9)}$.

Em relação ao agressor, na presente pesquisa, o/a filho/a apresentou maior incidência. Estudo realizado em três municípios brasileiros distintos apresentou como mais frequente o agressor do sexo masculino e membro da própria família ${ }^{(24)}$. Outro estudo identificou que a violência é comum entre idosos que dependem financeiramente do agressor ${ }^{(29)}$. Em uma análise por gênero, os filhos e parceiros conjugais eram os agressores das mulheres, enquanto os homens sofreram violência perpetrada por desconhecidos ${ }^{(30)}$.

Acerca do perfil de mortalidade por causa externas, na presente pesquisa, as quedas representaram os maiores incidentes de óbitos no estudo, que são eventos multifatoriais e influenciados pelas características ambientais e comportamentais ${ }^{(31)}$. Em uma análise da ocorrência de quedas em idosos, encontrou-se como as mais incidentes e associadas à população feminina, além de idade avançada, sedentarismo, pior percepção de saúde e quantidade de medicamentos ${ }^{(32)}$.

Considerando-se o conceito amplo de violência contra a pessoa idosa, as quedas podem estar relacionadas e serem subnotificadas quando há iatrogenia medicamentosa e precária infraestrutura de espaços públicos, caracterizado pela violência institucional. Além disso, o quadro de queda também predispõe à perda da funcionalidade e ao aumento da dependência após o episódio de queda ${ }^{(31)}$.

Os acidentes de trânsito são a segunda causa de morte no presente estudo. No Brasil, ocorreu uma elevação de $22,5 \%$ na taxa de mortalidade por acidentes de transportes terrestres no período de 2000 a 2010. Em 2000, a taxa era de 18,2 óbitos por 100 mil habitantes; em 2004, elevou-se para 20,3 e, em 2010, atingiu o valor de 22,3 óbitos ${ }^{(33)}$. Em análise do perfil das vítimas fatais nos acidentes de trânsito de 1996 a 2007, observa-se que a mortalidade de idosos era 2,5 vezes maior que na faixa etária de menores de 19 anos, com uma taxa média anual de $85,2 / 100$ mil habitantes ${ }^{(34)}$.

As agressões e os homicídios posicionam-se no quarto lugar quanto à causalidade dos óbitos no atual estudo, ocupando a mesma posição em estudos anteriores ${ }^{(17,21)}$. Em análise das internações hospitalares, outro estudo identificou que idosos do sexo masculino apresentam maior frequência de internações por agressão corporal ${ }^{(35)}$.

Em uma revisão sobre o suicídio para a população idosa, os pesquisadores identificaram que as mulheres idosas apresentaram maior tendência ao suicídio, enquanto a incidência dos óbitos é maior nos homens com a faixa etária a partir dos 80 anos, sendo a mais suscetível( ${ }^{(36)}$. Estudo realizado na cidade do Recife verificou maior incidência de casos entre os 60 e 69 anos de idade ${ }^{(37)}$. Uma pesquisa realizada com idosos que vivem em comunidade identificou que a depressão se apresenta como fator de risco para lesão autoprovocada e outros tipos de violência ${ }^{(26)}$.

Os eventos indeterminados representam a terceira provável causa de morte durante o período do estudo. Um estudo realizado em Minas Gerais expõe o crescimento da causa de mortalidade como evento de intenção indeterminada de 1999 a 2008, chegando a 18,4\% dos casos em 2008, indicando uma possível subnotificação de homicídios e outras causas de óbitos, além da má qualidade de preenchimento da Declaração de Óbito no estado ${ }^{(17)}$. Igualmente identificado no presente estudo, o qual também apresentou limitações referentes ao preenchimento das fichas de notificação, uma vez que campos estavam em branco ou ignorados. Um exemplo disso é a variável referente à escolaridade, que a maioria das fichas de notificação $(41,8 \%)$ apresentou como ignorados. 
Outra limitação é a subnotificação que pode ocorrer devido à ausência de procura de uma unidade de saúde (local onde são realizadas as notificações) por parte da vítima, associada com o possível despreparo dos profissionais de saúde para investigar os casos, devido à infraestrutura deficiente de atendimento e à fragilidade das redes de apoio ${ }^{(18)}$.

Contudo, existem políticas públicas para redução de agravos, garantia dos direitos e de proteção, e promoção de saúde à população idosa ${ }^{(20,38,39)}$. A Política Nacional de Redução da Morbimortalidade por Acidentes e Violência (PNRMAV), aprovada pela Portaria número 737/GM, de 16 de maio de 2001, por sua vez, objetiva reduzir a morbimortalidade por causas externas e instituir abordagem intersetorial, definindo concepções de ações que possibilitam a abordagem do estado no fenômeno da violência contra as pessoas idosas ${ }^{(38)}$.

Por sua vez, o Estatuto do Idoso, sancionado pela Lei $n .^{0} 10.741 / 2003$, formaliza em leis os seus direitos como qualquer outra pessoa humana, definindo as penalizações e tornando compulsória a notificação pelo profissional de saúde ${ }^{(39)}$.

A Política Nacional de Saúde da Pessoa Idosa, aprovada pela Portaria n. ${ }^{\circ} 2.528$, de 19 de outubro de 2006, direciona medidas coletivas e individuais de saúde para recuperar, manter e promover a autonomia e a independência dos indivíduos e amplia para a formação permanente dos profissionais, a cooperação internacional e o apoio à realização de estudos e pesquisas, além de abordar a intersetorialidade proposta pela PNRMAV( ${ }^{(2)}$.

Essas políticas públicas que se apresentam como consequência das prioridades gerais, do tempo histórico e dos problemas estruturais e conjunturais da sociedade orientam para a proteção e o cuidado com a pessoa e a garantia de direitos ${ }^{(40)}$.

A violência contra a pessoa idosa é um fenômeno multifatorial e complexo e conhecer a sua incidência e os fatores associados é útil no processo de promoção/prevenção da saúde da pessoa idosa. As notificações de violência, processo exigido por lei, e dados de mortalidade são ferramentas adequadas que permitem articulações e melhorias nos conjuntos de políticas públicas já existentes e a promoção de ações intersetoriais mais efetivas na defesa e proteção desse segmento populacional| ${ }^{(19)}$. A formação dos profissionais de saúde no tema e ações voltadas para a conscientização da população sobre o problema são necessárias para uma sociedade que envelhece ${ }^{(40)}$.

\section{CONCLUSÃO}

As principais vítimas da violência são as mulheres com baixa escolaridade, sendo decorrente de violência física perpetrada principalmente pelo/a filho/a, porém aqueles que vêm a óbito com mais frequência são os homens, tendo como principal causa as quedas e os acidentes de transporte.

\section{AGRADECIMENTOS E CONFLITOS DE INTERESSE}

Os autores agradecem a Luciana Karen Calabria pelo apoio e correção durante a construção do artigo. No presente estudo não há conflitos de interesses.

\section{CONTRIBUIÇÕES}

Rubens Correa Meirelles Junior, Julia de Oliveira Castro, Clarice Lima Álvares da Silva, Waneska Alexandra Alves contribuíram com a elaboração e delineamento do estudo; aquisição, análise e interpretação de dados; redação e revisão do manuscrito. Lina Rodrigues de Faria contribuiu com a elaboração e delineamento do estudo; redação e revisão do manuscrito.

\section{REFERÊNCIAS}

1. Dantas IC, Pinto EP Jr, Medeiros KKAS, Souza EA. Perfil de morbimortalidade e os desafios para a atenção domiciliar do idoso brasileiro. Rev Kairós [Internet]. 2017 [acesso em 2018 Mar 18];20(1):93-108. Disponível em:https://revistas.pucsp.br/index.php/kairos/article/view/32058.

2. Miranda GMD, Mendes ACG, Silva ALA. O envelhecimento populacional brasileiro: desafios e consequências sociais atuais e futuras. Rev Bras Geriatr Gerontol [Internet]. 2016 [acesso em 2018 Mar 20];19(3):507-19. Disponível em: http://www.scielo.br/pdf/rbgg/v19n3/pt_1809-9823-rbgg-19-03-00507.pdf.

3. Instituto Brasileiro de Geografia e Estatística. Pesquisa nacional por domicílio continuada [Internet]. Rio de Janeiro: IBGE; 2018 [acesso em 2019 Mar 21]. Disponível em: https://www.ibge.gov.br/estatisticasnovoportal/sociais/populacao/9171-pesquisa-nacional-por-amostra-de-domicilios-continua-mensal. $\mathrm{html} ?=\& \mathrm{t}=$ downloads. 
4. Instituto Brasileiro de Geografia e Estatística. Síntese de indicadores sociais [Internet]. Rio de Janeiro: IBGE; 2018 [acesso em 2019 Mar 21]. Disponível em: https://agenciadenoticias.ibge.gov.br/media/com_mediaibge/ arquivos/ce915924b20133cf3f9ec2d45c2542b0.pdf.

5. Alcântara AO, Camarano AA, Giacomin KC, organizadores. Política nacional do idoso: velhas e novas questões. Rio de Janeiro: Ipea; 2016.

6. Sanches APRA, Lebrão ML, Duarte YAO. Violência contra idosos: uma questão nova?. Saude Soc [Internet]. 2008 [acesso em 2018 Mar 20];17(3):90-100.

7. Organização Mundial da Saúde. Relatório mundial sobre a prevenção da violência. São Paulo: OMS; 2014.

8. Barcelos EM, Madureira MDS. Violência contra o idoso. In: Chaimowicz F, editor. Saúde do idoso. Belo Horizonte: UFMG; 2013, p. 132-41.

9. Yon $\mathrm{Y}$, Mikton $\mathrm{CR}$, Gassoumis ZD, Wilber KH. Elder abuse prevalence in community settings: a systematic review and meta-analysis. Lancet Glob Health [Internet]. 2017 [acesso em 2018 Out 5];5(2):e147-56. Disponível em: https://doi.org/10.1016/S2214-109X(17)30006-2.

10. Minayo MCS, Souza ER, Paula DR. Revisão sistemática da produção acadêmica brasileira sobre causas externas e violências contra a pessoa idosa. Ciênc Saúde Colet [Internet]. 2010 [acesso em 2018 Jun 20];15(6):2719-28. Disponível em: http://dx.doi.org/10.1590/S1413-81232010000600010.

11. Santos FS, Saintrain MVL, Vieira LJES, Sampaio EGM. Characterization and prevalence of elder abuse in Brazil. J Interpers Violence [Internet]. 2018 [acesso em 2019 Mar 29];00(0):1-17. Disponível em: https://doi.or g/10.1177\%2F0886260518781806.

12. Zakar R, Zakar MZ, Abbas S. Domestic violence against rural women in Pakistan: an issue of health and human rights. J Fam Violence [Internet]. 2016 [acesso em 2018 Out 5];31(1):15-25. Disponível em: https://doi. org/10.1007/s10896-015-9742-6.

13. Ministério da Saúde (BR). Departamento de Informática do SUS. Classificação Internacional de Doenças. $10^{a}$ ed. Brasília: Ministério da Saúde; 2015.

14. Gonsaga RAT, Rimoli CF, Pires EA, Zogheib FS, Fujino MVT, Cunha MB. Avaliação da mortalidade por causas externas. Rev Col Bras Cir [Internet]. 2012 [acesso em 2018 Abr 10];39(4):263-7. Disponível em: http://dx.doi.org/10.1590/S0100-69912012000400004

15. Minayo MCS, Souza ER. É possível prevenir a violência? Reflexões a partir do campo da saúde pública. Ciênc Saúde Colet [Internet]. 1999 [acesso em 2018 Abr 15];4(1):7-23. Disponível em: http://dx.doi. org/10.1590/S1413-81231999000100002

16. Secretaria de Direitos Humanos da Presidência da República (BR). Manual de enfrentamento à violência contra a pessoa idosa. É possível prevenir. É necessário superar. Brasília: Secretaria de Direitos Humanos da Presidência da República; 2013.

17. Cooper C, Livingston G. Intervening to reduce elder abuse: challenges for research. Age Ageing [Internet]. 2016 [acesso em 2018 Jun 6];45(2):184-5. Disponivel em: https://doi.org/10.1093/ageing/afw007.

18. Gomes LMX, Barbosa TLA, Caldeira AP. Mortalidade por causas externas em idosos em Minas Gerais, Brasil. Esc Anna Nery [Internet]. 2010 [acesso em 2018 Abr 15];14(4):779-86. Disponível em: http://dx.doi. org/10.1590/S1414-81452010000400018.

19. Rocha RC, Cortes MCJW, Dias EC, Gontijo ED. Violência velada e revelada contra idosos em Minas Gerais-Brasil: análise de denúncias e notificações. Saúde Debate [Internet]. 2018 [acesso em 2019 Mar 29]; 42(spe4):81-94. Disponível em: http://dx.doi.org/10.1590/0103-11042018s406.

20. Brasil. Ministério da Saúde. Portaria $n^{\circ} 2.528$, de 8 de outubro de 2006. Aprova a Política Nacional de Saúde da Pessoa Idosa [Internet]. 2006 [acesso em 2019 Mar 24]. Disponível em: http://bvsms.saude.gov.br/bvs/ saudelegis/gm/2006/prt2528_19_10_2006.html

21. Gawryszewski VP, Jorge MHPM, Koizumi MS. Mortes e internações por causas externas entre os idosos no Brasil: o desafio de integrar a saúde coletiva e atenção individual. Rev Assoc Med Bras [Internet]. 2004 [acesso em 2018 Out 18];50(1):97-103. Disponível em: http://dx.doi.org/10.1590/S0104-42302004000100044. 
22. Guedes DT, Curcio CL, Llano BA, Zunzunegui MV, Guerra R. La brecha de género en violencia doméstica en adultos mayores en América Latina: el Estudio IMIAS [The gender gap in domestic violence in older adults in Latin America: the IMIAS Study]. Rev Panam Salud Pública [Internet]. 2015 [acesso em 2018 Jun 8];37(4/5):293-300. Disponível em: https://www.scielosp.org/scielo.php?script=sci_arttext\&pid $=$ S1020-49892015000400016.

23. Hirt MC, Costa MC, Arboit J, Leite MT, Hesler LZ, Silva EB. Representações sociais da violência contra mulheres rurais para um grupo de idosas. Rev Gaúcha Enferm [Internet]. 2017 [acesso em 2018 Out 18];38(4):e68209. Disponível em: http://dx.doi.org/10.1590/1983-1447.2017.04.68209.

24. Rodrigues RAP, Monteiro EA, Santos AMR, Pontes MLF, Fhon JRS, Bolina AF, et al. Violência contra idosos em três municípios brasileiros. Rev Bras Enferm [Internet]. 2017 [acesso em 2018 Out 20];70(4):816-24. Disponível em: http://www.scielo.br/pdf/reben/v70n4/pt_0034-7167-reben-70-04-0783.pdf.

25. Alves WA, Faria L, Meirelle RCJ. Violência contra o idoso: uma realidade velada. In: Faria L, Calábria L, Alves W, organizadores. Envelhecimento: um olhar interdisciplinar. São Paulo: Editora Hucitec; 2016. p. 160-87.

26. Torres-Castro S, Szlejf C, Parra-Rodríguez L, Rosas-Carrasco O. Association between frailty and elder abuse in community-dwelling older adults in Mexico City. J Am Geriatr Soc [Internet]. 2018 [acesso em 2018 Out 5];66(9):1773-78. Disponível em: https://doi.org/10.1111/jgs.15489.

27. Oliveira MLC, Gomes ACG, Amaral CPM, Santos LB. Características dos idosos vítimas de violência doméstica no Distrito Federal. Rev Bras Geriatr Gerontol [Internet]. 2012 [acesso em 2018 Out 20];15(3):55566. Disponível em:http://www.scielo.br/pdf/csc/v17n8/30.pdf.

28. Duque AM, Leal MCC, Marques APO, Eskinazi FMV, Duque AM. Violência contra idosos no ambiente doméstico: prevalência e fatores associados (Recife/PE). Ciênc Saúde Colet [Internet]. 2012 [acesso em 2018 Jun 20];17(8):2199-208. Disponível em: http://www.scielo.br/pdf/rbgg/v15n3/v15n3a16.pdf.

29. Lacher S, Wettstein A, Senn O, Rosemann T, Hasler S. Types of abuse and risk factors associated with elder abuse. Swiss Med Wkly [Internet]. 2016 [acesso 2018 Set 10];146:w14273. Disponível em: https: https://smw. ch/article/doi/smw.2016.14273.

30. Mascarenhas MDM, Andrade SSCA, Neves ACM, Pedrosa AAG, Silva MMA, Malta DC. Violência contra a pessoa idosa: análise das notificações realizadas no setor saúde -Brasil. Ciênc Saúde Colet [Internet]. 2012 [acesso em 2018 Jun 20];17(9):2331-41. Disponível em: http://www.scielo.br/pdf/csc/v17n9/a14v17n9.pdf.

31. Nascimento CFD, Duarte YAO, Lebrão ML, Chiavegatto ADP Filho. Individual and neighborhood factors associated with functional mobility and falls in elderly residents of São Paulo, Brazil: a multilevel analysis. J Aging Health [Internet]. 2018 [acesso em 2018 Set 10];30(1):118-39. Disponível em: https://doi. org/10.1177\%2F0898264316669229.

32. Siqueira FV, Facchini LA, Piccini RX, Tomasi E, Thumé E, Silveira DS, et al. Prevalência de quedas em idosos e fatores associados. Rev Saúde Pública [Internet]. 2007 [acesso em 2018 Jun 25];41(5):749-56. Disponível em:http://www.scielo.br/pdf/rsp/v41n5/6188.pdf.

33. Morais OL Neto, Montenegro MMS, Monteiro RA, Siqueira JB Júnior, Silva MMA, Lima CM, et al. Mortalidade por acidentes de transporte terrestre no Brasil na última década: tendência e aglomerados de risco. Ciênc Saúde Colet [Internet]. 2012 [acesso em 2018 de Jun 25];17(9):2223-36. Disponível em: http://www.scielo.br/ $\mathrm{pdf} / \mathrm{csc} / \mathrm{v} 17 \mathrm{n} 9 / \mathrm{a} 02 \mathrm{v} 17 \mathrm{n} 9 . \mathrm{pdf}$.

34. Camargo FC, Hemiko H. Vítimas fatais e anos de vida perdidos por acidentes de trânsito em Minas Gerais, Brasil. Esc Anna Nery [Internet]. 2012 [acesso em 2018 de Jun 25];16(1):141-6. Disponível em: http://www. scielo.br/pdf/ean/v16n1/v16n1a19.pdf.

35. Castro VC, Rissardo LK, Carreira L. Violência contra os idosos brasileiros: uma análise das internações hospitalares. Rev Bras Enferm [Internet]. 2018 [acesso em 25 de Jun 2018];71(suppl 2):830-8. Disponível em: http://www.scielo.br/pdf/reben/v71s2/pt_0034-7167-reben-71-s2-0777.pdf.

36. Minayo MCS, Cavalcante FG. Tentativas de suicídio entre pessoas idosas: revisão de literatura (2002/2013). Ciênc Saúde Colet [Internet]. 2015 [acesso em 2018 Jun 25];20(6):1751-62. Disponível em: http://www.scielo. $\mathrm{br} / \mathrm{pdf} / \mathrm{csc} / \mathrm{v} 20 \mathrm{n} 6 / 1413-8123-\mathrm{csc}-20-06-1751$.pdf. 
37. Mendonça FA Neto, Melo AAG, Queiroz AFB, Paiva SOC, Lima FM. Suicídio em idosos no Recife (PE): um estudo sobre mortalidade por causas externas. Rev Kairós [Internet]. 2014 [acesso em 2018 Jun 25];16(5):255-67. Disponível em: https://revistas.pucsp.br/index.php/kairos/article/view/18647.

38. Ministério da Saúde (BR). Política Nacional de Redução da Morbimortalidade por Acidentes e Violência. Brasília: Ministério da Saúde; 2001.

39. Brasil. Lei $n^{\circ} 10.741$, de 1 de outubro de 2003. Dispõe sobre o Estatuto do Idoso e dá outras providências. Diário Oficial da União; Brasília, 1 Out 2003.

40. Moreira WC, Damasceno CKCS, Vieira SKSF, Campêlo TPT, Campêlo DS, Alencar DC. Análise sobre as políticas públicas de enfrentamento a violência contra o idoso. Rev Enferm [Internet]. 2016 [acesso em 2019 Mar 29];10(4):1324-31. Disponível em: https://periodicos.ufpe.br/revistas/revistaenfermagem/article/ download/11120/12601.

\section{Endereço do primeiro autor:}

Rubens Correa Meirelles Junior

Universidade Federal de Juiz de Fora - UFJF

Campus Avançado de Governador Valadares

Rua Manoel Byrro, 241

Bairro: Vila Bretas

CEP: 35032-620 - Governador Valadares - MG - Brasil

E-mail: rubens.correamjr@outlook.com

\section{Endereço para correspondência:}

Waneska Alexandra Alves

Universidade Federal de Juiz de Fora - UFJF

Campus Avançado de Governador Valadares

Rua Manoel Byrro, 241

Bairro: Vila Bretas

CEP: 35032-620- Governador Valadares - MG - Brasil

E-mail: waneska2812@hotmail.com

Como citar: Meirelles RC Junior, Castro JO, Faria LR, Silva CLA, Alves WA. Notificações de óbitos por causas externas e violência contra idosos: uma realidade velada. Rev Bras Promoç Saúde. 2019;32:8685. 